\title{
Analog and RF Circuit Macromodels for System-Level Analysis
}

\author{
Xin Li, Peng Li, Yang Xu, Lawrence T. Pileggi \\ Dept. of ECE, Carnegie Mellon University \\ 5000 Forbes Avenue, Pittsburgh, PA 15213, USA \\ \{xinli, pli, yangx, pileggi\}@ece.cmu.edu
}

\begin{abstract}
Design and validation of mixed-signal integrated systems require system-level model abstractions. Generalized Volterra series based models have been successfully applied for analog and RF component macromodels, but their complexity can sometimes limit their utility for time-varying systems and large circuits with complex device models or numerous parasitics. In this paper we propose simple and efficient analog and RF circuit macromodels that provide accurate model abstractions for large, complex timevarying circuits over frequency bands of interest. By starting with the system-level block diagram model structures and focusing on the narrow RF bands, the proposed macromodels can efficiently capture the nonlinear behavior as well as the impact of RLC coupling parasitics via compact reduced-order model forms. While the macromodel can trade accuracy for simplicity in terms of the number of frequency expansion points, we find that expansion about one frequency point provides the accuracy required for system-level analysis of most RF and narrow-band analog components. The macromodel form corresponds to block diagram structures that are easily incorporated into our systemlevel simulation tool based on Simulink.
\end{abstract}

\section{Categories and Subject Descriptors}

B.7.2 [Integrated Circuits]: Design Aids-Verification.

\section{General Terms}

Algorithms

\section{Keywords}

Macromodel, Analog/RF Circuits

\section{INTRODUCTION}

The advance of VLSI technology has made it possible to integrate an entire mixed-signal communication system onto a single IC or within a single electronic package. To design such systems affordably requires a top-down design methodology that includes efficient exploration of system-level architectures before detailed circuit implementation. In addition, following the design implementation, bottom-up verification is required to validate the overall system performance with the inclusion of nonidealities and parasitics. Clearly, both top-down design and bottom-up

Permission to make digital or hard copies of all or part of this work for personal or classroom use is granted without fee provided that copies are not made or distributed for profit or commercial advantage and that copies bear this notice and the full citation on the first page. To copy otherwise, or republish, to post on servers or to redistribute to lists, requires prior specific permission and/or a fee.

DAC 2003, June 2-6, 2003, Anaheim, California, USA.

Copyright 2003 ACM 1-58113-688-9/03/0006...\$5.00. verification require model abstractions of the RF/analog components that are compatible with system-level analyses.

During the past decade, Volterra series models have been developed for characterizing RF/analog circuits [1]-[3]. These macromodeling approaches have a solid theoretical background [4] and can be extremely accurate and efficient for small circuit blocks. In addition, the Volterra series model in symbolic form provides intuitive information on the internal behavior of $\mathrm{RF} /$ analog circuits. However, the complexity of the Volterra series model grows dramatically as the circuit size increases, since order reduction issue is not addressed in [1]-[3]. Even for small circuits, adding parasitic components can result in complicated Volterra series models. For this reason, the Volterra series model in its symbolic form is most applicable for top-down design/synthesis at early design stages [5], where parasitic components are not included.

To address the model order reduction problem for analog components with parasitics, research has evolved from the interconnect macromodeling work that has been primarily focused on linear time-invariant (LTI) systems [6]-[8]. Recently, these techniques were extended to time-varying systems and weakly nonlinear systems in [9]-[12]. The nonlinear order reduction algorithms proposed in [10]-[12] are mathematically elegant and can generate accurate reduced-order systems for general nonlinear circuits over a wide frequency range, although they result in somewhat complex model representations.

As IC technologies are scaled to finer feature sizes and circuit applications move to higher frequency bands, the problem of efficient RF component macromodeling becomes more pronounced. First, device models become increasingly complex in order to characterize the physical behavior for small devices at high frequency [13]. The high order derivative information that is required for some of the macromodeling approaches [1]-[3], [10][11] is not explicitly available for the complex semi-empirical device models, such as the BSIM3 model. Secondly, as the frequency increases, the parasitics become more important and more complex. However, the bandwidths for GSM and WCDMA receivers are $935 \sim 960 \mathrm{MHz}$ and $2110 \sim 2170 \mathrm{MHz}$ [14]-[15] respectively, for example. In most system-level simulation tools such as SPW (Cadence) and COSSAP (Synopsys), complex lowpass representation is employed to represent and simulate the narrow-band signal flow in analog and mixed-signal systems. These commercial system-level simulators, consequently, require macromodels for specific frequency bands, rather than a general wide-band model.

In this paper we propose a macromodeling methodology for analog and RF components over a narrow-band frequency range of interest. Importantly, since the effect of frequency translation appears in both nonlinear systems and linear periodically timevarying (LPTV) systems, we analyze the nonlinearities via a 
linear time-varying approach. This, in turn, indicates that, within a narrow bandwidth, the frequency domain Volterra kernel transfer functions can be approximated accurately by applying existing linear order reduction algorithms for a corresponding LPTV system at the center frequency (expansion point). Next, we demonstrate that these narrow-band Volterra kernel transfer functions can be mapped to simple block diagram model structures required for system-level simulation. Theoretically, by increasing the number of center frequencies (expansion points) for Volterra kernel transfer function extraction, the proposed macromodeling approach can be applied for complicated model structures containing static nonlinear functions and linear transfer functions. In this paper, we find that simple model structure associated with one expansion point provides the sufficient accuracy within the narrow band of interest, even if the original circuit nonlinearity is a complex function over a wide frequency range.

The proposed macromodeling approach, ROMAN (ReducedOrder Macromodeling of Analog including Nonlinearities), provides several promising features compared with other modeling approaches. First, no high order derivative information of nonlinear devices is required during model generation. Second, the macromodel transfer functions are simplified by existing order reduction algorithms for linear systems. As such, simple macromodels are produced without requiring special nonlinear order reduction techniques. Finally, taking advantage of the narrow-band property, the proposed macromodel structures are assembled via simple block diagrams, and, consequently, are easily incorporated into a system-level simulation tool based on Simulink. The accuracy and efficiency of the proposed macromodels in system-level simulation are demonstrated by a GSM receiver in $0.25 \mu \mathrm{m}$ CMOS process.

\section{BACKGROUND}

\subsection{Volterra Series Theory}

In general, the Volterra series representation of a timeinvariant nonlinear system can be written as [4]:

$$
y(t)=\sum_{n=1}^{N} y_{n}(t)
$$

where $y_{n}(t)$ is the nth order response given by

$$
y_{n}(t)=\int_{-\infty}^{+\infty} \cdots \int_{-\infty}^{+\infty} h_{n}\left(\tau_{1}, \cdots, \tau_{n}\right) \cdot x\left(t-\tau_{1}\right) \cdot x\left(t-\tau_{n}\right) \cdot d \tau_{1} \cdots d \tau_{n}
$$

$x(t)$ and $y(t)$ are system input and output respectively, $h_{n}\left(\tau_{1}, \cdots, \tau_{n}\right)$ is the nth order Volterra kernel, and $N$ is the maximum order of system nonlinearities.

Volterra series theory also provides an explicit formula for the steady-state response of weakly nonlinear systems. Consider the excitation of a multi-tone input

$$
x(t)=\sum_{i=1}^{R}\left|A_{i}\right| \cos \left(\omega_{i} t+\angle A_{i}\right)=\sum_{i=-R, i \neq 0}^{R} \frac{A_{i}}{2} e^{j \omega_{i} t}
$$

where $A_{-i}=A_{i}^{*}$ denotes the conjugate of $A_{i}$, and $\omega_{-i}=-\omega_{i}$, then the nth order steady-state response of the system can be described as

$$
\begin{aligned}
y_{n}(t) & =\frac{1}{2^{n}} \sum_{i 1=-R, i 1 \neq 0}^{R} \cdots \sum_{i n=-R, i n \neq 0}^{R} A_{i 1} \cdots A_{i n} \\
& \cdot H_{n}\left(j \omega_{i 1}, \cdots, j \omega_{i n}\right) \cdot e^{j\left(\omega_{i 1}+\cdots+\omega_{i n}\right) \cdot t}
\end{aligned}
$$

where

$$
\begin{aligned}
H_{n}\left(j \omega_{1}, \cdots, j \omega_{n}\right) & =\int_{-\infty}^{+\infty} \cdots \int_{-\infty}^{+\infty} h_{n}\left(\tau_{1}, \cdots, \tau_{n}\right) \\
& \cdot e^{-j\left(\omega_{1} \tau_{1}+\cdots+\omega_{n} \tau_{n}\right)} \cdot d \tau_{1} \cdots d \tau_{n}
\end{aligned}
$$

is the nth order kernel transfer function. Without loss of generality, we assume the Volterra kernel transfer functions are represented by their symmetric forms in this paper.

\subsection{Linear Periodically Time-varying System}

Consider a nonlinear system described by a set of differentialalgebraic equations [10]

$$
\begin{gathered}
\frac{d q[\hat{x}(t)]}{d t}+f[\hat{x}(t)]=b(t)+B \cdot u(t) \\
\hat{y}(t)=D \cdot \hat{x}(t)
\end{gathered}
$$

where $\hat{x}(t)$ are the time-domain circuit variables or unknowns, $b(t)$ is a vector of large-signal excitations, and $q(\bullet)$ and $f(\bullet)$ are nonlinear functions describing the charge/flux and resistive terms, respectively, in the circuit. $B$ and $D$ are vectors that link the smallsignal input $u(t)$ and output $\hat{y}(t)$ to the rest of the system.

If input $u(t)$ is small, its effect can be characterized by a perturbation analysis. Let $x^{*}(t)$ denote the large-signal solution of (6) with $u(t)$ set to zero. Linearizing (6) at $x^{*}(t)$ yields

$$
\begin{gathered}
C(t) \cdot \frac{d x(t)}{d t}+G(t) \cdot x(t)=B \cdot u(t) \\
y(t)=D \cdot x(t)
\end{gathered}
$$

where $x(t)$ and $y(t)$ are the small-signal versions of $\hat{x}(t)$ and $\hat{y}(t)$ respectively, and $C(t)$ and $G(t)$ are the time-varying derivative matrices of $q(\bullet)$ and $f(\bullet)$.

Equation (7) describes a linear time-varying system with input $x(t)$ and output $y(t)$. Especially, if the matrix coefficients $C(t)$ and $G(t)$ in (7) are periodic in time, such a system is so called linear periodically time-varying (LPTV) system. The input-output relation of the LPTV system can be described by a frequencydomain transfer function [10], [11]

$$
Y(t, j \omega)=\hat{H}(t, j \omega) \cdot X(j \omega)
$$

where

$$
\begin{gathered}
X(j \omega)=\int x(t) \cdot e^{-j \omega t} \cdot d t \\
y(t)=\frac{1}{2 \pi} \int Y(t, j \omega) \cdot e^{j \omega t} \cdot d \omega
\end{gathered}
$$

For an LPTV system with period $T$, the time-varying transfer function $\hat{H}(t, j \omega)$ in (8) is periodic in $t$, and, therefore, can be expanded into Fourier series [10]

$$
\hat{H}(t, j \omega)=\sum_{k=-K}^{K} \hat{H}_{k}(j \omega) \cdot e^{j k \omega_{0} t}
$$

where $\hat{H}_{k}(j \omega)$ is the Fourier coefficient, $\omega_{0}=2 \pi / T$ and $K$ is large enough so that the truncation error of the Fourier expansion in (10) is negligible. Substitute (10) into (8), then the following equation gives the steady-state response of the LPTV system under multi-tone input in (3).

$$
y(t)=\sum_{i=-R, i \neq 0}^{R} \sum_{k=-K}^{K} \frac{A_{i}}{2} \cdot \hat{H}_{k}\left(j \omega_{i}\right) \cdot e^{j\left(\omega_{i}+k \omega_{0}\right) t}
$$




\section{MACROMODELING FOR WEAKLY NONLINEAR TIME-INVARIANT SYSTEM}

\subsection{Linear Time-Varying Analysis}

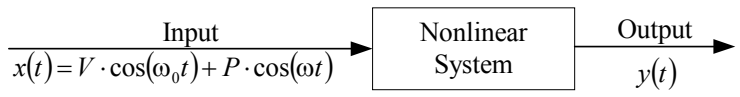

Fig. 1. Input-output relation of a nonlinear system

It is well known that both nonlinear system and LPTV system present the effect of frequency translation. In what follows, we study the circuit nonlinearities from the perspective of linear timevarying analysis. As such, we can derive a relation between the Volterra series representation and the LPTV system representation. For the reason of simplicity, we assume that the circuit for macromodeling is weakly nonlinear and its maximum order of nonlinearities is equal to 3 .

Consider a weakly nonlinear system with a two-tone input $V \cdot \cos \left(\omega_{0} t\right)+P \cdot \cos (\omega t)$, as shown in Fig. 1. Both $V$ and $P$ are small enough so that the system in Fig. 1 remains weakly nonlinear. If we regard $V \cdot \cos \left(\omega_{0} t\right)$ as the input excitation that determines the time-varying operation point of the nonlinear circuit, then the input-output relation from input $P \cdot \cos (\omega t)$ to output $y(t)$ can be described by an LPTV system. As such, the steady-state output response $y(t)$ can be evaluated based on the LPTV system equations summarized in (8)-(11).

$$
\begin{gathered}
y(t)=\bar{y}_{1}(t)+\bar{y}_{2}(t)+\bar{y}_{3}(t) \\
\bar{y}_{1}(t)=\frac{P}{2} \cdot\left[\hat{H}_{0}(j \omega) \cdot e^{j \omega t}+\hat{H}_{0}(-j \omega) \cdot e^{-j \omega t}\right] \\
\bar{y}_{2}(t)=\frac{P}{2} \cdot \sum_{k= \pm 1}\left[\hat{H}_{k}(j \omega) \cdot e^{j \omega t}+\hat{H}_{k}(-j \omega) \cdot e^{-j \omega t}\right] \cdot e^{j k \omega_{0} t} \\
\bar{y}_{3}(t)=\frac{P}{2} \cdot \sum_{k= \pm 2}\left[\hat{H}_{k}(j \omega) \cdot e^{j \omega t}+\hat{H}_{k}(-j \omega) \cdot e^{-j \omega t}\right] \cdot e^{j k \omega_{0} t}
\end{gathered}
$$

Equation (14) and (15) show that the output response $y(t)$ presents frequency translation effect since it contains harmonic components at various frequencies $\left\{\omega+k \omega_{0} ; k= \pm 1, \pm 2\right\}$. Such a frequency translation is referred as the intermodulation effect in the community of nonlinear circuit analysis [1], if the nonlinear system in Fig. 1 is studied under the two-tone input $V \cdot \cos \left(\omega_{0} t\right)+P \cdot \cos (\omega t)$. In the following, we show that the LPTV response in (12)-(15) is actually a subset of the overall response generated by the nonlinear circuit. Based on Volterra series theory described in (1)-(5), output $y(t)$ is given by

$$
\begin{gathered}
y(t)=\tilde{y}_{1}(t)+\tilde{y}_{2}(t)+\tilde{y}_{3}(t) \\
\tilde{y}_{1}(t)=\frac{1}{2} \cdot \sum_{m=-2, m \neq 0}^{2} A_{m} H_{1}\left(j \omega_{m}\right) \cdot e^{j \omega_{m} t} \\
\tilde{y}_{2}(t)=\frac{1}{4} \cdot \sum_{m=-2, m \neq 0}^{2} \cdot \sum_{n=-2, n \neq 0}^{2} A_{m} A_{n} H_{2}\left(j \omega_{m}, j \omega_{n}\right) \cdot e^{j\left(\omega_{m}+\omega_{n}\right) \cdot t} \\
\tilde{y}_{3}(t)=\frac{1}{8} \cdot \sum_{m=-2, m \neq 0}^{2} \cdot \sum_{n=-2, n \neq 0}^{2} \cdot \sum_{l=-2, l \neq 0}^{2} A_{m} A_{n} A_{l} \\
\cdot H_{3}\left(j \omega_{m}, j \omega_{n}, j \omega_{l}\right) \cdot e^{j\left(\omega_{m}+\omega_{n}+\omega_{l}\right) \cdot t}
\end{gathered}
$$

where

$$
\begin{gathered}
A_{-1}=A_{1}=V \quad A_{-2}=A_{2}=P \\
\omega_{-1}=-\omega_{0} \quad \omega_{1}=\omega_{0} \quad \omega_{-2}=-\omega \quad \omega_{2}=\omega
\end{gathered}
$$

After some mathematical manipulations, $y(t)$ can be partitioned into two parts

$$
y(t)=y_{L}(t)+y_{N L}(t)
$$

In (22), $y_{L}(t)$ denotes the linear response, namely which is linearly proportional to the sinusoidal input amplitude $P$,

$$
\begin{gathered}
y_{L}(t)=\tilde{y}_{L 1}(t)+\widetilde{y}_{L 2}(t)+\widetilde{y}_{L 3}(t) \\
\tilde{y}_{L 1}(t)=\frac{P}{2} \cdot\left[H_{1}(j \omega) \cdot e^{j \omega t}+H_{1}(-j \omega) \cdot e^{-j \omega t}\right] \\
\tilde{y}_{L 2}(t)=\frac{P V}{2} \cdot \sum_{k= \pm 1}\left[H_{2}\left(j \omega, j k \omega_{0}\right) \cdot e^{j \omega t}\right. \\
\left.+H_{2}\left(-j \omega, j k \omega_{0}\right) \cdot e^{-j \omega t}\right] \cdot e^{j k \omega_{0} t} \\
\tilde{y}_{L 3}(t)=\frac{3 P V^{2}}{8} \cdot \sum_{k= \pm 1}\left[H_{3}\left(j \omega, j k \omega_{0}, j k \omega_{0}\right) \cdot e^{j \omega t}\right. \\
\left.+H_{3}\left(-j \omega, j k \omega_{0}, j k \omega_{0}\right) \cdot e^{-j \omega t}\right] \cdot e^{j 2 k \omega_{0} t} \\
+\frac{3 P V^{2}}{4} \cdot\left[H_{3}\left(j \omega, j \omega_{0},-j \omega_{0}\right) \cdot e^{j \omega t}\right. \\
\left.+H_{3}\left(-j \omega, j \omega_{0},-j \omega_{0}\right) \cdot e^{-j \omega t}\right]
\end{gathered}
$$

and $y_{N L}(t)$ denotes the nonlinear response that is a nonlinear function of $P$. As a subset of the overall response generated by the nonlinear circuit, $y_{L}(t)$ in (23)-(26) gives the output response that is linearly proportional to input $P \cdot \cos (\omega t)$. Therefore, it is exactly the output response evaluated by the LPTV analysis in (12)-(15). Comparing (12)-(15) with (23)-(26), we reach the following relations.

$$
\begin{gathered}
\hat{H}_{0}(s)=H_{1}(s)+\frac{3 V^{2}}{2} \cdot H_{3}\left(s, j \omega_{0},-j \omega_{0}\right) \\
\left\{\begin{array}{l}
\hat{H}_{1}(s)=V \cdot H_{2}\left(s, j \omega_{0}\right) \\
\hat{H}_{-1}(s)=V \cdot H_{2}\left(s,-j \omega_{0}\right)
\end{array}\right. \\
\left\{\begin{array}{l}
\hat{H}_{2}(s)=\frac{3 V^{2}}{4} \cdot H_{3}\left(s, j \omega_{0}, j \omega_{0}\right) \\
\hat{H}_{-2}(s)=\frac{3 V^{2}}{4} \cdot H_{3}\left(s,-j \omega_{0},-j \omega_{0}\right)
\end{array}\right.
\end{gathered}
$$

where $s=j \omega$. (27)-(29) are the key equations connecting original circuit nonlinearities with the corresponding LPTV system. Note that the LPTV transfer functions $\hat{H}_{ \pm 1}(s)$ and $\hat{H}_{ \pm 2}(s)$ can be easily extracted by existing linear order reduction techniques [9], [10]. Substituting $\hat{H}_{ \pm 1}(s)$ and $\hat{H}_{ \pm 2}(s)$ into (28)(29), consequently, results in reduced-order Volterra kernels $H_{2}\left(s, \pm j \omega_{0}\right)$ and $H_{3}\left(s, \pm j \omega_{0}, \pm j \omega_{0}\right)$. On the other hand, the reduced-order transfer function $H_{1}(s)$ can be easily extracted by linearizing the original nonlinear circuit at the DC operation point, although (27) doesn't give an explicit relation between $\hat{H}_{0}(s)$ and $H_{1}(s)$.

\subsection{Model Structure Mapping}

The Volterra kernel transfer functions $H_{1}(s), H_{2}\left(s, \pm j \omega_{0}\right)$ and $H_{3}\left(s, \pm j \omega_{0}, \pm j \omega_{0}\right)$ determine the circuit behaviors near the center frequency (expansion point) $\omega_{0}$. In this subsection, we show that these kernel transfer functions can be mapped to simple macromodel structures. 


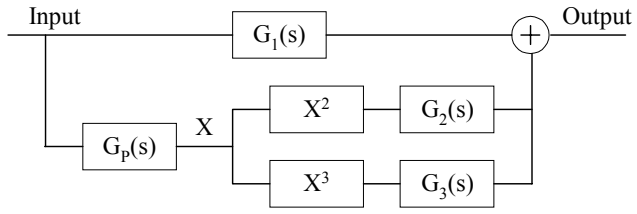

Fig. 2. A simple macromodel structure

Fig. 2 shows a simple model structure that is an extension of the Hammerstein model in [16]. In this subsection, we use this model structure as an example for Volterra kernel mapping, although the proposed ROMAN algorithm is not restricted to specific macromodel structures. For the macromodel in Fig. 2,

$$
G_{1}(s)=H_{1}(s)
$$

is the 1st order Volterra kernel transfer function, and its 2nd and 3rd order Volterra kernel transfer functions are given by [4]

$$
\begin{gathered}
H_{2}\left(s_{1}, s_{2}\right)=G_{P}\left(s_{1}\right) \cdot G_{P}\left(s_{2}\right) \cdot G_{2}\left(s_{1}+s_{2}\right) \\
H_{3}\left(s_{1}, s_{2}, s_{3}\right)=G_{P}\left(s_{1}\right) \cdot G_{P}\left(s_{2}\right) \cdot G_{P}\left(s_{3}\right) \cdot G_{3}\left(s_{1}+s_{2}+s_{3}\right)
\end{gathered}
$$

Substitute $s_{1}=s$ and $s_{2}=s_{3}= \pm j \omega_{0}$ into (31)-(32), and, after some mathematical manipulations, we obtain

$$
\begin{gathered}
G_{2}(s)=\frac{\sum_{k= \pm 1} H_{2}\left(s-j k \omega_{0}, j k \omega_{0}\right)}{\sum_{k= \pm 1} G_{P}\left(j k \omega_{0}\right) \cdot G_{P}\left(s-j k \omega_{0}\right)} \\
G_{3}(s)=\frac{\sum_{k= \pm 1} H_{3}\left(s-j 2 k \omega_{0}, j k \omega_{0}, j k \omega_{0}\right)}{\sum_{k= \pm 1} G_{P}^{2}\left(j k \omega_{0}\right) \cdot G_{P}\left(s-j 2 k \omega_{0}\right)}
\end{gathered}
$$

The detailed proof for (33)-(34) can be found in [17]. In (33)-(34), $G_{P}(s)$ represents the transfer function of the input impedance match network employed in most RF circuits, and it can be easily extracted by applying linear order reduction algorithms at the DC operation point. Then, after $G_{P}(s), H_{1}(s), H_{2}\left(s, \pm j \omega_{0}\right)$ and $H_{3}\left(s, \pm j \omega_{0}, \pm j \omega_{0}\right)$ are determined, the transfer functions $G_{1}(s), G_{2}(s)$ and $G_{3}(s)$ in Fig. 2 are determined as well.

A simple version of the ROMAN algorithm is summarized in Fig. 3. The simple ROMAN algorithm in Fig. 3 approximates the Volterra kernel transfer functions $H_{2}\left(s, \pm j \omega_{0}\right)$ and $H_{3}\left(s, \pm j \omega_{0}, \pm j \omega_{0}\right)$ at one center frequency (expansion point) $\omega_{0}$. However, our complete ROMAN algorithm is not restricted to such a single-point expansion. If necessary, we can extract the reduced-order Volterra kernel transfer functions at multi center frequencies (expansion points), i.e. $\left\{H_{2}\left(s, \pm j \omega_{i}\right), i=1,2, \cdots\right\}$ and $\left\{H_{3}\left(s, \pm j \omega_{i}, \pm j \omega_{i}\right), i=1,2, \cdots\right\}$, and map those kernels to more complicated model structures. As the number of expansion points $\omega_{i}$ increases, we can include more problem unknowns into the model structure and, theoretically, the ROMAN algorithm can be applied to various macromodels containing static nonlinear functions and linear transfer functions. The essence of the ROMAN algorithm is to externally approximate the nonlinear circuit behavior within the selected frequency bands of interest. However, as the bandwidth increases, a great number of frequency expansion points, as well as complicated model structures, are required to achieve the sufficient accuracy. In such cases, the ROMAN algorithm might lose its efficiency, since it doesn't explore the internal structure of the circuit and solving a large set of problem unknowns externally results in high computation cost and various numerical problems. The Volterra series approaches in [1]-[3], on the other hand, investigate each nonlinearity in the circuit and build the macromodel internally. These approaches can capture circuit behaviors over a wide frequency range with complex macromodels, although the privilege of selecting frequency bands is lost during their internal macromodeling process.

- Compute the DC operation point of the nonlinear circuit and extract the reduced-order transfer function $H_{1}(s)$ and $G_{P}(s)$ by PRIMA [8].

- Given a sinusoidal input $V \cos \left(\omega_{0} t\right)$, compute the steadystate solution $x^{*}(t)$ of the nonlinear circuit. Here, $\omega_{0}$ is the center frequency and $V$ is chosen small enough so that the circuit remains weakly nonlinear up to 3 rd order.

- Linearize the nonlinear circuit at $x^{*}(t)$ and extract the reduced-order LPTV transfer function $\hat{H}_{ \pm 1}(s)$ and $\hat{H}_{ \pm 2}(s)$ by TVP [10].

- Compute the reduced-order Volterra kernel transfer function $H_{2}\left(s, \pm j \omega_{0}\right)$ and $H_{3}\left(s, \pm j \omega_{0}, \pm j \omega_{0}\right)$ based on equation (28)-(29).

- Map the obtained $H_{1}(s), \quad H_{2}\left(s, \pm j \omega_{0}\right)$ and $H_{3}\left(s, \pm j \omega_{0}, \pm j \omega_{0}\right)$ to the model structure in Fig. 2 using equation (30), (33)-(34).

Fig. 3. Simple ROMAN implementation

\section{MACROMODELING FOR WEAKLY NONLINEAR TIME-VARYING SYSTEM}

The ROMAN algorithm proposed in this paper can be extended to weakly nonlinear periodically time-varying (NLPTV) systems, such as the RF mixer with fixed LO input. Note that, for NLPTV macromodeling, the symbols $G_{1}(s), G_{2}(s)$ and $G_{3}(s)$ in

Fig. 2 represent the corresponding LPTV transfer functions. Due to the limit of space, the detailed mathematic description for NLPTV macromodeling is not included here. Further discussions on this topic can be found in [17].

\section{SIMULATION RESULTS}

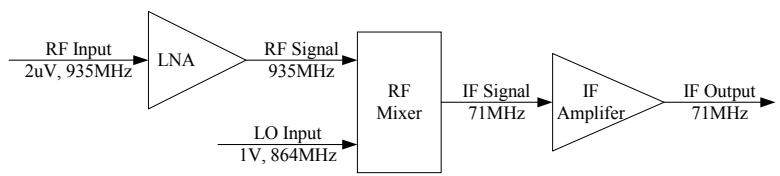

Fig. 4. Block diagram of GSM receiver

Shown in Fig. 4 is a GSM receiver system in $0.25 \mu \mathrm{m}$ TSMC CMOS process. Employing the proposed ROMAN algorithm, we first build the macromodels for LNA and RF mixer. The IF amplifier in Fig. 4 is assumed to be ideal for this analysis and, therefore, it is modeled by a linear transfer function. We should note, however, that nothing precludes us from representing this component by a ROMAN macromodel as well. Next, we connect all these components together and simulate the overall GSM receiver in our simulation tool to verify its performance at the system level. All the simulations are performed on a Pentium IV$1.4 \mathrm{GHz}$ computer with $256 \mathrm{MB}$ of memory. 


\subsection{Low Noise Amplifier}

Fig. 5 shows the circuit schematic of a differential LNA that includes 13 MOS transistors. This unusually large number of transistors for an LNA is a good example since it produces highly complex nonlinear behaviors to test the proposed macromodels. The center frequency of the LNA is $900 \mathrm{MHz}$. Applying ROMAN, we construct the LNA macromodel by the simple structure in Fig. 2. In this example, $G_{P}(s)$ is the linear transfer function of the input impedance match network, as illustrated in Fig. 5. TABLE I shows the order numbers for both the original circuit and the linear transfer functions in the macromodel.

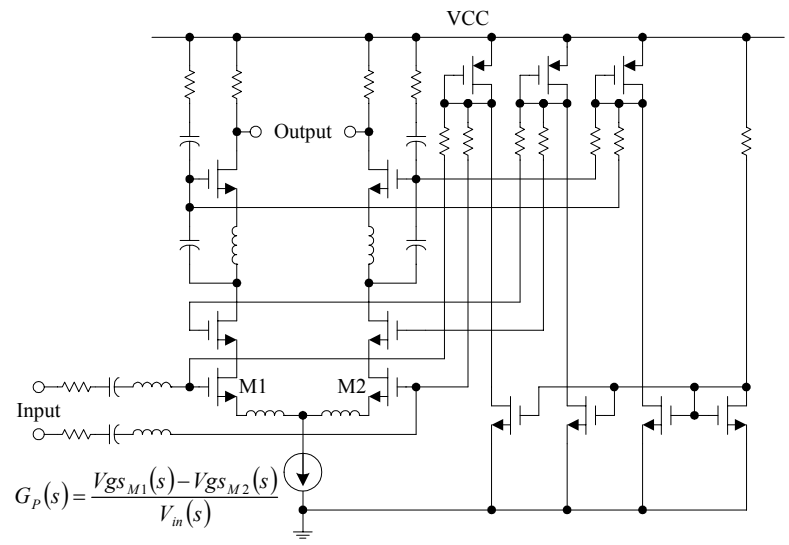

Fig. 5. Circuit schematic of LNA

TABLE I. System order for LNA macromodel

\begin{tabular}{|c|c|c|c|c|c|}
\hline & Original Circuit & $\overline{G_{P}(s)}$ & $G_{1}(s)$ & $G_{2}(s)$ & $\overline{G_{3}(s)}$ \\
\hline Order & 39 & 6 & 4 & 8 & 8 \\
\hline
\end{tabular}
macromodel using single-tone input with an amplitude range of $1 \sim 10 \mathrm{mV}$ and frequency range of $750 \sim 1050 \mathrm{MHz}$. This frequency range is reasonably large compared with typical bandwidths for RF applications [14]-[15]. Even for such a wide bandwidth, the maximum modeling error is $2.27 \%$ when defined as:

$$
\text { Error }=\sqrt{\left[\sum_{i=-N}^{N}\left|\hat{Y}_{i}-Y_{i}\right|^{2}\right] /\left[\sum_{i=-N}^{N}\left|\hat{Y}_{i}\right|^{2}\right]}
$$

In (35), $\hat{Y}_{i}$ is the ith order output harmonic obtained by the original LNA circuit, $Y_{i}$ is the corresponding result estimated by the macromodel, and $N$ is chosen large enough so that those high order harmonics beyond $N$ are negligible.

Next, we run a two-tone test on the developed macromodel. The amplitude and frequency ranges of the 1st sinusoidal tone are set to $1 \sim 10 \mathrm{mV}$ and $750 \sim 1050 \mathrm{MHz}$ respectively. The amplitude of the 2 nd sinusoidal tone is fixed at $10 \mathrm{mV}$, and the frequency difference between two input tones is fixed at $1 \mathrm{MHz}$. Fig. 6 shows the estimated 3rd order intermodulation distortion (the only in-band distortion for LNA) from the macromodel. Fig. 7 shows the corresponding absolute error for the estimated distortion.

Two observations can be made based on the error distribution in Fig. 7. First, the developed macromodel is most accurate around the center frequency $900 \mathrm{MHz}$, since, in this example, the reduced-order Volterra kernel transfer functions $H_{2}\left(s, \pm j \omega_{0}\right)$ and $H_{3}\left(s, \pm j \omega_{0}, \pm j \omega_{0}\right)$ are extracted at the center frequency $\omega_{0}=2 \pi \cdot 900 \mathrm{Rad} / \mathrm{Sec}$. This observation indicates that the frequency bands of interest can be selected by the ROMAN algorithm during the macromodeling process. Second, but more importantly, the high macromodeling accuracy at the center frequency $900 \mathrm{MHz}$ implies that, although the overall nonlinearities for the original LNA circuit are complicated in this example, its narrow-band behavior can still be approximated accurately by a simple macromodel structure associated with a single frequency expansion point.

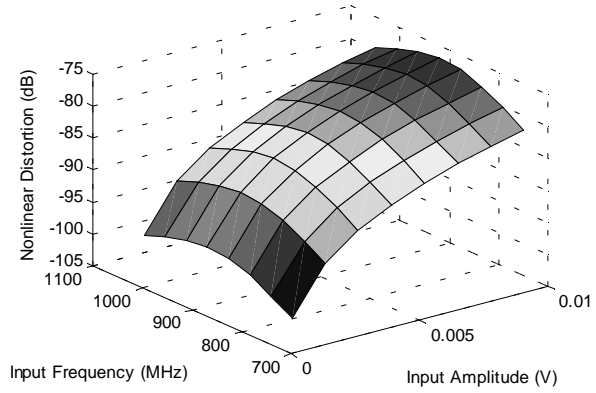

Fig. 6. Estimated distortion from macromodel

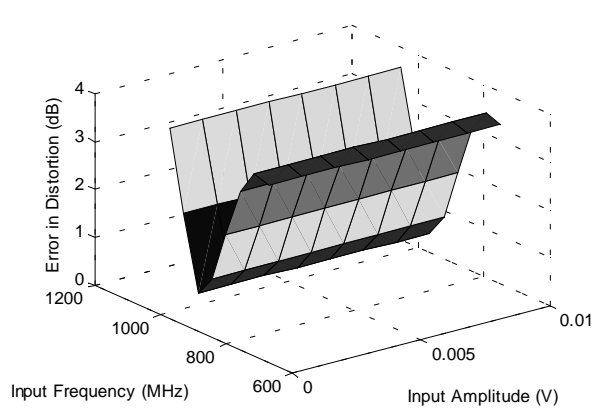

Fig. 7. Error in intermodulation distortion

For testing the simulation speed of the macromodel, we perform steady-state simulation on both the LNA circuit and its macromodel under single-tone input. The computation costs are 1.71 seconds for the original circuit and 0.0293 seconds for the macromodel respectively.

\subsection{Double-Balanced Mixer}

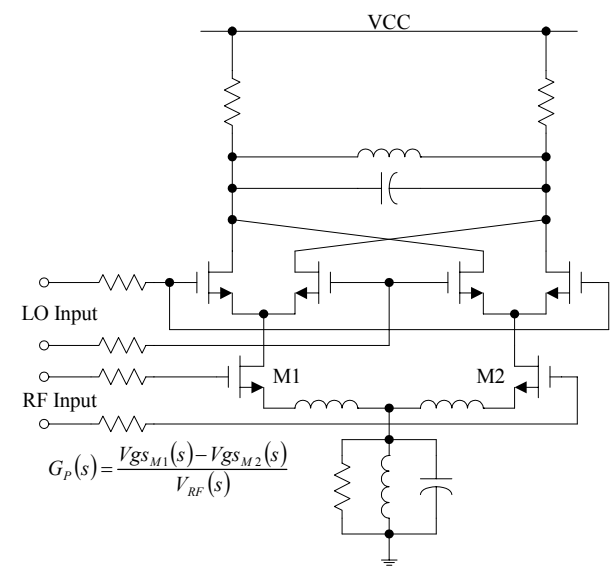

Fig. 8. Circuit schematic of mixer

Fig. 8 displays a double-balanced mixer for down-conversion, in which the LO input is fixed as a sinusoidal signal of amplitude $2 \mathrm{~V}$ and frequency $874 \mathrm{MHz}$. The input-output behavior from RF 
input to IF output, therefore, is a weakly nonlinear periodically time-varying system [3]. We build the mixer model using the model structure in Fig. 2. TABLE II shows the order numbers for both the original circuit and the linear transfer functions in the macromodel ${ }^{*}$.

TABLE II. System order for mixer macromodel

\begin{tabular}{c|c|c|c|c|c}
\hline & Original Circuit & $G_{P}(s)$ & $G_{1}(s)$ & $G_{2}(s)$ & $G_{3}(s)$ \\
\hline Order & 345 & 11 & 11 & 16 & 16 \\
\hline
\end{tabular}

We run steady-state analysis to test the macromodeling accuracy where the amplitude and frequency ranges for the RF input are set to $10 \sim 300 \mathrm{mV}$ and $750 \sim 1050 \mathrm{MHz}$ respectively. The maximum modeling error is $7.23 \%$. Here, the macromodeling error is similarly evaluated using (35).

For testing the simulation speed of the mixer model, we run steady-state simulation on both the mixer circuit and its macromodel. The computation costs are 10954 seconds for the original circuit and 0.0765 seconds for the macromodel respectively.

\subsection{System-Level Simulation}
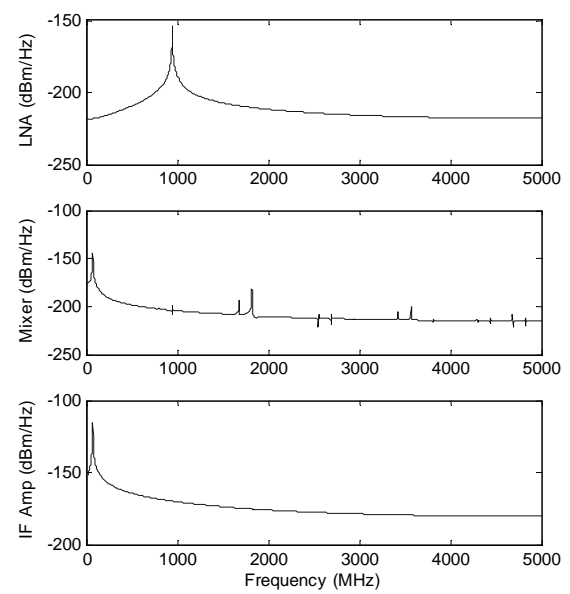

Fig. 9. Simulation results for GSM receiver

Using our system-level simulation tool based on Simulink, a transient simulation is performed for the overall GSM receiver for the time range $[0,1 \mu s]$. An FFT is applied to the output signal after the steady state is reached. Fig. 9 displays the output frequency spectra of various circuit components. The overall computation time is 28.44 seconds. Note that, without such macromodels, system-level verification with the inclusion of parasitics becomes impractical.

\section{CONCLUSIONS}

The behavior of RF circuits is characterized by complicated nonlinearities, due to the numerous nonlinear components that contribute to the overall circuit performance. However, efficient high-level simulation of RF systems requires simple macromodels for individual circuit blocks. By exploring the narrow-band property in RF applications, the proposed ROMAN algorithm can generate simple macromodels for RF circuits within the selected

\footnotetext{
${ }^{*}$ In this case, mixer is a periodically time-varying system. Its order is proportional to the harmonic number generated by the LO excitation [10]. Due to the large LO amplitude, the LO harmonic number is set to 15 in this example.
}

frequency bands of interest. These simple macromodels, with block diagram structures, can be easily incorporated into our system-level simulation tool based on Simulink for efficient highlevel evaluation, as demonstrated by the GSM receiver system example.

\section{ACKNOWLEDGMENTS}

This work has been supported by the MARCO Center for Circuits, Systems and Software (under MARCO contract 2001CT-888 and DARPA grant MDA972-02-1-0004) and the Semiconductor Research Corporation (under contract 2000-TJ779).

\section{REFERENCES}

[1] P. Wambacq and W. Sansen, Distortion Analysis of Analog Integrated Circuits, Kluwer Academic Publishers, Boston, 1998.

[2] P. Wambacq, P. Dobrovolny, S. Donnay, M. Engels and I. Bolsens, "Compact modeling of nonlinear distortion in analog communication circuits," IEEE DATE, pp. 350-354, 2000.

[3] I. Vassiliou and A. Sangiovanni-Vincentelli, "A frequency-domain, Volterra series-based behavioral simulation tool for RF systems," IEEE CICC, pp. 21-24, 1999.

[4] M. Schetzen, The Volterra and Wiener Theories of Nonlinear Systems, J. Wiley \& Sons, 1980.

[5] G. Gielen, "Modeling and analysis techniques for system-level architectural design of telecom front-ends," IEEE Trans. MTT, vol. 50, pp. 360-368, Jan. 2002.

[6] L. T. Pillage and R. A. Rohrer, "Asymptotic waveform evaluation for timing analysis," IEEE Trans. CAD, vol. 9, pp. 352-366, Apr. 1990.

[7] P. Feldmann and R. W. Freund, "Efficient linear analysis by Pade approximation via Lanczos process," IEEE Trans. CAD, vol. 14, pp. 639-649, May. 1995.

[8] A. Odabasioglu, M. Celik and L. T. Pileggi, "PRIMA: passive reduced-order interconnect macromodeling algorithm," IEEE Trans. $C A D$, vol. 17, pp. 645-654, Aug. 1998.

[9] J. Phillips, "Model reduction of time-varying linear systems using approximate multipoint krylov-subspace projectors," IEEE ICCAD, pp. 96-102, 1998.

[10] J. Roychowdhury, "Reduced-order modeling of time-varying systems," IEEE Trans. CAS - II, vol. 26, pp. 1273-1288, Oct. 1999.

[11] J. Phillips, "Projection frameworks for model reduction of weakly nonlinear systems," IEEE DAC, pp. 184-189, 2000.

[12] M. Rewienski and J. White, "A trajectory piecewise-linear approach to model order reduction and fast simulation of nonlinear circuits and micromachined devices," IEEE ICCAD, pp. 252-257, 2001.

[13] M. Chan, K. Hui, C. Hu and P. Ko, "A robust and physical BSIM3 nonquasistatic transient and $\mathrm{AC}$ small-signal model for circuit simulation," IEEE Trans. Electron Devices, vol. 45, pp. 834-841, Apr. 1998.

[14] P. Orsatti, F. Piazza and Q. Huang, "A 20mA-receiver, 50-mAtransmit, single-chip GSM transceiver in $0.25-\mu \mathrm{m}$ CMOS," IEEE JSSSC, vol. 34, pp. 1869-1879, Dec. 1999.

[15] A. Parssinen, J. Jussila, J. Ryynanen, L. Sumanen and K. Halonen, "A 2-GHz wide-band direct conversion receiver for WCDMA applications," IEEE JSSC, vol. 34, pp. 1893-1903, Dec. 1999.

[16] H. Unbehauen and G. Rao, Identification of Continuous Systems, Elsevier Science Publishing Inc., 1987.

[17] X. Li, P. Li, Y. Xu and L. Pileggi, "Analog and RF circuit macromodels for system-level analysis," Technical Report, Carnegie Mellon University, 2002. 